\title{
Estudo comparativo dos métodos de simulação SNESIM e FILTERSIM - aplicado a um modelo sintético de cobre
}

Comparative study of SNESIM and FILTERSIM simulations methods - applied to a copper synthetic model

\author{
EDUARDO HENRIQUE DE MORAES TAKAFUJI ${ }^{1}$; MARCELO MONTEIRO ROCHA ${ }^{1}$; GUSTAVO \\ ZANCO RAMOS ${ }^{1}$
}

1'Universidade de São Paulo - USP, São Paulo-eduardo.takafuji@usp.br,mmrocha@usp.br,gustavo.ramos@usp.br

\begin{abstract}
Resumo
Os métodos de simulação geoestatística tradicionais podem apresentar resultados inadequados quando estruturas complexas como boudins, dobras, falhas, canais meandrantes, dentre outras, precisam ser reproduzidas. Pois, para modelar estruturas complexas, o uso do variograma é limitado e para suprir esta limitação, os métodos geoestatísticos de multiponto foram propostos. Neste trabalho é apresentado um estudo comparativo em que os algoritmos de simulação multiponto (Multiple-Point Simulation - MPS) SNESIM e FILTERSIM são aplicados à um depósito sintético de cobre. Este depósito foi criado como análogo a um depósito real para ser utilizado como referência. Ele mimetiza uma mineralização complexa que implica em alta variabilidade dos padrões geológicos e, portanto, a simulação multiponto foi realizada em um cenário também complexo. Os métodos simulam os padrões encontrados em uma imagem de treinamento (IT), que deve ter geologia análoga a do local estudado. As simulações foram calculadas utilizando três malhas amostrais diferentes com amostras coletadas a aproximadamente cada $60 \mathrm{~m}, 40 \mathrm{~m}$ e, por fim, $20 \mathrm{~m}$. Considerando as duas amostragens mais dispersas, os resultados obtidos pela simulação FILTERSIM apresentam melhor conectividade espacial, sendo visualmente mais representativos da região simulada e comparando suas estatísticas globais com as amostras e a imagem de treinamento, elas são mais representativas que as estatísticas obtidas pelo SNESIM.
\end{abstract}

Palavras-chave: Simulação Multiponto; SNESIM; FILTERSIM; Geoestatística; Cobre.

\begin{abstract}
Traditional geostatistical simulation methods may present inadequate results when complex structures such as boudins, folds, faults, meandering channels, among others, need to be reproduced. In order to model complex structures, the use of the variogram can be limited and to overcome this limitation, geostatistical multiple-point methods have been proposed. This comparative study applies two multiple-point simulation algorithms, the SNESIM and FILTERSIM, to a synthetic copper deposit. This deposit was created analogous to an actual deposit to be used as a reference. It mimics a complex mineralization that implies high variability of the geological patterns and, therefore, the multiple-point simulation was carried out in a complex scenario. The methods simulate the patterns found in a training image (IT), which should have an analogous geology of the studied site. The simulations were calculated using three different sample meshes with samples collected at approximately every $60 \mathrm{~m}, 40 \mathrm{~m}$ and, finally, $20 \mathrm{~m}$. Considering the two most dispersed samplings, the results obtained by the FILTERSIM simulation show better spatial connectivity, being visually more representative of the simulated region and comparing its global statistics with the samples and the training image, they are more representative than the statistics obtained by SNESIM.
\end{abstract}

Keywords: Multiple-point simulation; SNESIM; FILTERSIM; Geostatistics; Copper.

\section{Introdução}

Strebelle (2002), Zhang et al. (2006), Dimitrakopoulos et al. (2010), entre outros, descrevem que os métodos de simulação por estatística multiponto (Multiple-Point Statistics - MPS) utilizam estatísticas de alta-ordem (média, variância, assimetria, curtose e outras) por meio de uma imagem de treinamento. Esta imagem é um banco de dados de padrões, que pode ser um modelo geológico ou geofísico. A imagem deve representar as características geológicas da área de estudo em sua totalidade. Portanto, é necessário haver uma biblioteca de padrões geológicos ou a modelagem completa de região análoga que emprestará seus padrões à região a ser simulada. A imagem de treinamento é utilizada para capturar a essência dos padrões geológicos apresentados na região (por exemplo, as principais relações geométricas que formam o modelo geológico do local). Quanto maior a 
complexidade do depósito, mais amostras serão necessárias, além de uma imagem de treinamento mais detalhada. O método SNESIM (Single Normal Equation SIMulation) simula apenas variáveis categóricas e, a fim de reproduzir os padrões, ele varre a imagem de treinamento calculando a probabilidade de ocorrência de cada um dos padrões possíveis, que são guardados em árvores de busca. $\mathrm{O}$ método, FILTERSIM, varre a imagem para capturar todos os padrões e suas probabilidades de ocorrência, estes padrões são guardados em classes, cuja dimensões são previamente definidas de acordo com o tamanho das estruturas a serem evidenciadas, por fim padrões semelhantes são agrupados. As imagens de treinamento podem ser obtidas em um banco de dados ou, criadas em um programa de modelagem geológica tridimensional, como Datamine $\AA$ ou Leapfrog $\AA$.

Escolheu-se trabalhar com um banco de dados sintético, para poder estudar a qualidade dos resultados destes métodos de simulação multiponto. Esta escolha deve-se, principalmente, a dois motivos:

I) $\mathrm{O}$ modelo geológico da mineralização é baseado em um depósito real e os teores de cobre foram definidos a partir de um depósito análogo. Portanto, eles são suscetíveis à variação natural das categorias a serem simuladas. Neste trabalho foram simuladas três amostragens do depósito;

II) Por ser uma amostragem dentro de depósito sintético, é possível comparar os resultados com a geologia "real".

O depósito sintético foi dividido em duas regiões, a porção sul tem a sequência estratigráfica definida e será utilizada como imagem de treinamento. Nos 300 metros da porção norte foram realizadas amostragens em 3 malhas regulares variando o distanciamento entre as sondagens em 60, 40 e 20 metros. Os litotipos são três, meta-arenito intercalado com filito, dobrados e cortados por uma falha inversa preenchida por veios silicosos com cobre associado.

Este trabalho tem como objetivo a aplicação e comparação dos algoritmos de simulação de multipontos SNESIM e FILTERSIM, ambos disponíveis no programa Stanford Geostatistical Modeling Software (SGeMS). Para tal, foram realizados exaustivos testes a fim de verificar a sensibilidade de cada parâmetro de entrada. Posteriormente, fez-se a comparação da qualidade e eficácia destes algoritmos em relação a representatividade espacial dos resultados aferida pela reprodução da proporção de cada litologia nos resultados.

\section{Métodos}

\subsection{Simulação de multipontos}

Caers \& Zhang (2002) explicam que a geoestatística tradicional baseada em semivariogramas é inadequada, uma vez que o semivariograma é muito limitado para capturar a heterogeneidade geológica dos dados, já a técnica intitulada de multipontos não utiliza o semivariograma, mas $\operatorname{sim}$ estruturas denominadas imagens de treinamento. As imagens de treinamento podem representar estruturas geológicas complexas e curvilíneas - como regiões com muitas dobras e falhas ou simplesmente canais meandrantes - melhor que os métodos geoestatísticos baseados em semivariogramas, que não capturam esta complexidade matematicamente. A simulação por estatística de multipontos captura os padrões espaciais das imagens de treinamento e os reproduz nos resultados. Apesar de não utilizar o variograma como ferramenta, estes métodos utilizam os mesmos princípios da geoestatística tradicional, que são: variáveis estacionárias de segunda ordem e ergodicidade nas estatísticas.

Segundo Journel \& Zhang (2006), a estatística de multipontos vai muito além da estatística convencional (histograma) e da estatística de dois pontos (covariograma). Ela pode simular realizações com alta entropia - regiões de geologia complexa e grande heterogeneidade geológica e de teores - enquanto oferece flexibilidade por considerar diversas imagens de treinamento alternativas com vários níveis de estruturas geológicas mais simples, ou seja, de baixa entropia.

Entre os principais algoritmos de multipontos estão: ENESIM (Extended Normal Equation Simulation) proposto por Guardiano \& Srivastava (1993), SNESIM (Single Normal Equation Simulation) apresentado por Strebelle (2002), SIMPAT (Simulation with Patterns) proposto por Arpat (2005) e Arpat \& Caers (2005), FILTERSIM (Filter-based Simulation) apresentado por Zhang et al. (2006), WAVESIM (Wavelet-based Simulation) apresentado por Gloaguen \& Dimitrakopoulos (2009), DISTPAT (Distance-based Pattern Simulator) mostrado por Honarkhah \& Caers (2010), DS (Direct Sampling) por Mariethoz et al. (2010) e HOSIM (High-Order Simulation) proposto por Dimitrakopoulos et al. (2010). Atualmente os mais utilizados são o SNESIM e o FILTERSIM, disponíveis no programa SGeMS.

De acordo com Caers \& Zhang (2002) e Journel \& Zhang (2006), as imagens de treinamento são, essencialmente, um banco de dados de padrões geológicos a partir do qual as estatísticas de vários pontos - incluindo o semivariograma - podem ser emprestadas. Ao simular em um domínio finito, devese seguir os princípios de ergodicidade e estacionariedade, deste modo, deve ficar claro que meras fotografias, desenhos de afloramentos ou sistemas de deposição não podem ser utilizados como imagens de treinamento se não seguirem esses princípios básicos da geoestatística tradicional. Ortiz (2003) explica que a reprodução da imagem de treinamento (IT) não é o objetivo, mas sim a captura dos padrões geológicos que serão reproduzidos e, assim, pode-se simular um modelo que compartilhe as características multivariadas da distribuição real.

Lyster et al. (2004) relatam que a imagem de treinamento deve ser considerada inteiramente representativa da área a ser simulada e deve conter todas as suas características geológicas. Para tanto, 
Caers \& Zhang (2002) explicam que a imagem de treinamento substitui o semivariograma como medida de heterogeneidade geológica e é mais intuitiva, uma vez que é possível observar - antes de qualquer simulação ou estimativa geoestatística - quais serão os padrões a serem reproduzidos. É mais fácil recusar uma imagem de treinamento baseada em uma inspeção ou interpretação visual da realidade geológica do que recusar um modelo de semivariograma ou suposição multigaussiana. Mais informações e aplicações dos métodos de multipontos podem ser encontradas em Mariethoz \& Caers (2014).

\subsection{SNESIM}

Segundo Caers \& Zhang (2002), o conceito de sequencialidade na simulação utilizado no algoritmo da SNESIM é semelhante àquele dos métodos de simulação geoestatística, como na simulação

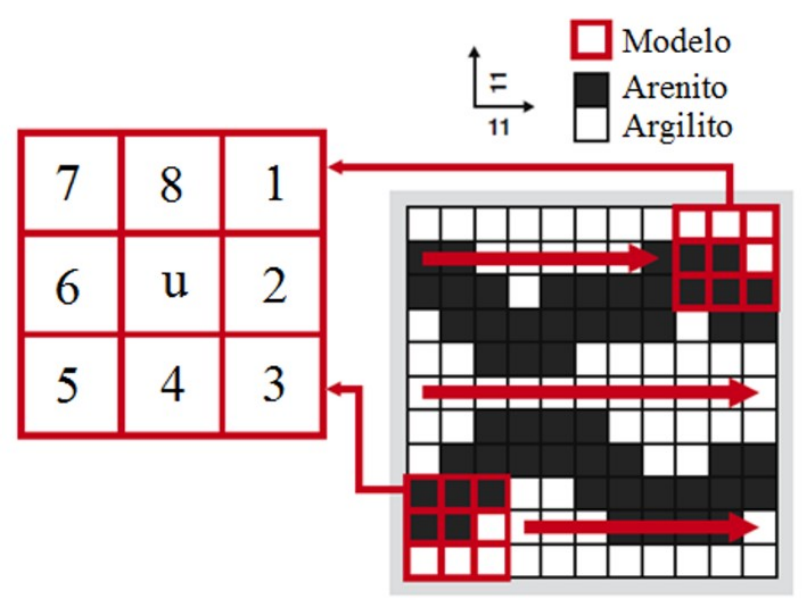

sequencial gaussiana. Ele se baseia na ideia de simular, sequencialmente, cada célula da malha ao longo de um caminho aleatório e inclui o valor simulado como dado condicionante para a simulação dos nós seguintes.

Segundo Vries et al. (2009), o algoritmo SNESIM é baseado em recuperar as estatísticas espaciais das imagens de treinamento, escaneando cada imagem com as configurações de pontos, chamado modelo (Figura 1), e as probabilidades de ocorrência de cada ponto $\mathrm{u}+\mathrm{hx}$ - sendo $\mathrm{hx}$ o índice de cada local ao redor do ponto estudado $u$, são guardadas em uma árvore de busca (Figura 2). Cada nível da árvore de busca representa mais informação no modelo. Durante a simulação, quanto mais informação espacial, mais níveis estarão presentes na árvore de busca e, consequentemente, mais preciso será o resultado. A simulação pode ser realizada em diferentes escalas, ou seja, a imagem de treinamento e a área a ser simulada não precisam estar na mesma escala.

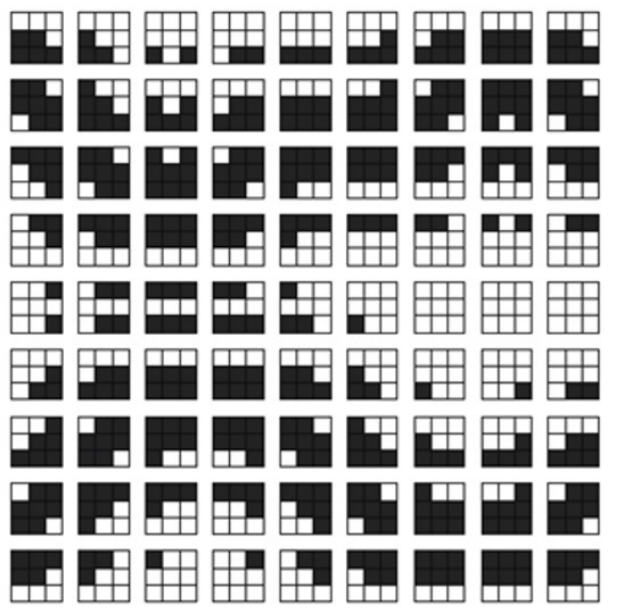

Figura 1 - Definição do modelo, exemplo de um modelo 2D 3x3 em uma malha 11 x 11 e seus padrões obtidos pelo escaneamento de um mapa onde a célula vazia representa a presença de argilito e a célula preenchida a presença de arenito (modificado de Arpat \& Caers 2007).

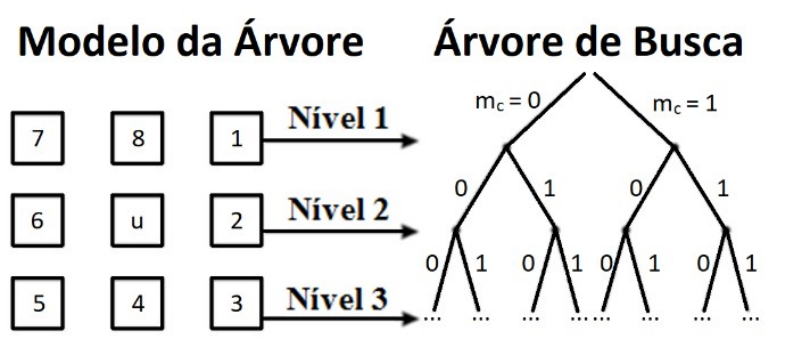

Figura 2 - Árvore de Busca com duas categorias $\left(\mathrm{m}_{\mathrm{c}}=0\right.$ ou $\left.\mathrm{m}_{\mathrm{c}}=1\right) \mathrm{e}$ modelo relacionado. Apenas os primeiros três locais $\left(\mathrm{h}_{\mathrm{j}}\right)$ no modelo são exibidos nesta árvore de busca (Vries et al. 2009). O dado condicionante, $\mathrm{u}$, representa o chamado nível 0 da árvore de busca.

Para garantir a reprodução dos dados amostrados nos respectivos locais, toda a amostragem original deve ser relocada para o nó mais próximo e seus valores fixados. A pequena escala da continuidade espacial da imagem de treinamento é passada às realizações simuladas através das proporções de treinamento, por isso, uma vez que o nó, ao ser simulado, se aproxima da localização de um dado amostrado, a variância condicional diminui de acordo com as imagens de treinamento (Strebelle 2002).
Remy et al. (2011) apresentam no programa SGeMS o algoritmo para a simulação pelo método SNESIM conforme:

-Escolher o número NG de malhas múltiplas;

-Começar com a malha mais grossa $\mathrm{Gg}, \mathrm{g}=\mathrm{NG}$

Enquanto $\mathrm{g}>0$ :

-Recolocar os dados amostrais no nó mais próximo da malha;

-Construir um modelo de busca;

-Construir uma árvore de busca usando a imagem de treinamento e o modelo de busca do item anterior;

-Definir um caminho aleatório para visitar todos os nós a serem simulados;

Para cada nó não amostrado do caminho aleatório:

-Encontrar os eventos dos dados condicionantes definidos no modelo de busca;

-Recuperar a distribuição da probabilidade condicional da árvore de busca;

-Sortear um valor simulado a partir da probabilidade condicional e adicioná-lo ao conjunto de dados;

-Recolocar todos os dados amostrais no seu local de origem se $\mathrm{g}>1$; 


\author{
-Mover para a malha mais fina $\mathrm{Gg}-1$ (considere $\mathrm{g}=\mathrm{g}$ \\ $-1)$
}

Ainda de acordo com Vries et al. (2009), a árvore de busca permite reduzir drasticamente o tempo utilizado para a recuperação das frequências, porque a árvore é constante em cada escala de simulação e só precisa ser calculada uma vez. A estrutura em árvore é criada por adição de todos os valores, recuperados pela aplicação do modelo a cada local da imagem de treinamento. $\mathrm{O}$ primeiro nó, nível 0 da árvore, corresponde à localização u. Cada nível seguinte da árvore corresponde a uma posição $h_{j}$ no modelo da árvore (Figura 2). Um nó se ramifica em $\mathrm{C}$ novos nós, sendo $\mathrm{C}$ o número total de possíveis categorias. O nó possuí, para diferentes categorias $\mathrm{m}_{\mathrm{c}}$, a correspondente frequência de ocorrência dos valores no local u até o respectivo nível. Cada adição de um local no modelo significa que a árvore é expandida a um nível extra. $\mathrm{O}$ tamanho máximo da árvore é $\mathrm{n}_{\max }=\mathrm{CJ}$, onde $\mathrm{J}$ é o número de níveis.

Como descrito em Vries et al. (2009), durante o processo de simulação, as probabilidades são recuperadas da árvore de busca usando uma lista de valores de $\mathrm{V}(\mathrm{u})$. Se a combinação de todos valores ocorrer na imagem de treinamento, a frequência para cada categoria pode ser encontrada no nó do último nível da árvore. Se esta combinação nunca ocorrer, o último valor na lista $\mathrm{V}(\mathrm{u})$ é retirado e uma nova lista $\mathrm{V}(\mathrm{u})$, com um elemento a menos, será recuperada da árvore. Este processo é aplicado iterativamente até uma correspondência ou até que todos os elementos da lista sejam retirados. Neste último caso, a distribuição de probabilidade marginal - a distribuição de probabilidade quando não há informações adicionais disponíveis - será utilizada para gerar um novo valor.

Os parâmetros de entrada utilizados para calcular as 100 realizações estocásticas das três categorias de litotipos pelo SNESIM utilizaram a semente (gerador de dados aleatórios): 211175 , distribuição marginal de 0,8; 0,15; 0,05 (Filito=0; Meta-Arenito=1; Quartzito $=2$, respectivamente). O modelo de busca utiliza 90 nós, seu alcance máximo é de 150 , o intermediário é 100 e o mínimo 75 , enquanto a rotação utilizada é $0^{\circ}$ em $\mathrm{Z}, 0^{\circ} \mathrm{em} \mathrm{X} \mathrm{e} 45^{\circ} \mathrm{em} \mathrm{Y}$. Além disso foi utilizado no mínimo uma repetição, fator de servo-system (influência da proporção alvo no resultado) de 0,5 , limiar de ressimulação (valor mínimo de vizinhos ausentes para haver ressimulação) de 5, uma malha múltipla e 3 interações de ressimulação. Para esta simulação, o modelo foi isotrópico e não foram utilizadas proporções verticais, dados indiretos, rotações, redimensionamento e sub-malha.

\subsection{FILTERSIM}

Segundo $\mathrm{Wu}$ et al. (2008), neste método os padrões de treinamento extraídos de imagens são identificados por um vetor utilizando alguns filtros de distribuições de valores. Estes padrões são então classificados e agrupados em classes de padrões. Cada classe é resumida pelo padrão médio ou protótipo. Ao invés de salvar todas as probabilidades em árvores de frequência - como é feito no SNESIM de Strebelle (2002) - o FILTERSIM salva apenas a identificação do padrão das imagens de treinamento.

Segundo Journel \& Zhang (2006), o FILTERSIM não exige muita memória RAM para realizar a simulação, pois reduz as dimensões com a aplicação de filtros ( 6 em imagens bidimensionais e 9 em imagens tridimensionais). Esta técnica trabalha a imagem de treinamento cortando-a em pedaços, como um quebracabeça, do tamanho do modelo de digitalização e em seguida classificam-se as peças semelhantes em classes de padrões. A varredura da imagem de treinamento é análoga a descrita para a SNESIM (Figura 1). A simulação procede como uma reconstrução de um "quebra-cabeça" e as peças (classes de padrões) que melhor corresponder às amostras fixadas e as peças vizinhas já simuladas são utilizadas na imagem que está sendo construída. As peças deste quebra-cabeça nunca se esgotam, pois, qualquer pedaço retirado é imediatamente substituído por outro idêntico.

Do ponto de vista probabilístico, pode-se considerar essas pilhas ou classes de padrões semelhantes como a distribuição dos padrões de multipontos construídos a partir da imagem de treinamento. A simulação consiste, primeiro, em recuperar dados condicionantes que informam a vizinhança local a ser simulada, em seguida, procurar a classe que melhor corresponda a esses dados e, por último, sortear de um padrão para a classe ou distribuição condicional (Journel \& Zhang 2006).

Basicamente o algoritmo FILTERSIM começa decompondo os padrões da imagem em poucos filtros de distribuições lineares e, a partir das médias ponderadas dos valores da imagem de treinamento sobre uma janela-móvel de um tamanho específico, são designados pesos para estas distribuições. Os padrões da imagem de treinamento são classificados nestes filtros no espaço multidimensional e essa classificação, por sua vez, é feita uma vez por imagem de treinamento, antes do processo de simulação e visa o agrupamento de padrões similares, onde a similaridade é definida por uma medida específica de distância (Journel \& Zhang 2006).

A simulação sequencial prossegue ao longo de um caminho aleatório para visitar os nós não amostrados da malha. Em cada nó o dado condicionante disponível dentro do modelo de vizinhança está relacionado com a classe de treinamento mais próxima a ele, de acordo com a distância anterior. Um padrão de treinamento é desenhado a partir dessa classe e corrigido para a malha de simulação centrada no nó visitado. O padrão desenhado consiste de um modelo de valores em pontos, cujos centros (são fixados para não serem ressimulados, ou seja, essas posições são removidas do caminhamento aleatório de visita. Dados amostrais são condicionados através do congelamento dos correspondentes nós da malha, portanto nunca serão alterados durante o curso de uma simulação. Estes 
dados contribuem, preferencialmente, para as distâncias de similaridade, garantindo, assim, que nenhum padrão de treinamento seja mantido se criadas descontinuidades (Journel \& Zhang 2006).

As etapas do algoritmo mostrado por Remy et al. (2011), para o FILTERSIM são:

-Criar mapas de distribuições com os filtros redimensionados;

-Particionar os padrões de treinamento em classes e seus protótipos;

-Recolocar os dados amostrais no nó mais próximo da malha;

-Definir um caminho aleatório para visitar todos os nós a serem simulados nesta malha;

Para cada nó não amostrado do caminho aleatório:

-Extrair o evento do dado condicionante;

-Encontrar o protótipo primário mais próximo deste evento;

Se o protótipo tiver listas de protótipos secundários então:

-Encontrar os protótipos secundários mais próximos do evento;

-Escolher aleatoriamente um padrão do protótipo secundário;

Se não

-Escolher aleatoriamente um padrão do protótipo primário;

-Fixar o padrão na realização sendo simulada e adicioná-lo ao banco de dados

Foram calculadas 100 realizações estocásticas por FILTERSIM com semente (gerador de sequência de dados aleatórios) inicial igual a 211175. O tamanho do modelo 3D utilizado como modelo de busca para definição dos filtros foi $11 \mathrm{~m}$ para direção $\mathrm{x} ; 11 \mathrm{~m}$ para y e $5 \mathrm{~m}$ para $\mathrm{z}$ e a dimensão do modelo é $7 \mathrm{~m}$ para direção $\mathrm{x} ; 7 \mathrm{~m}$ para y e $3 \mathrm{~m}$ para $\mathrm{z}$. Estes valores foram escolhidos para que, em média, haja ao menos 2 amostras no plano horizontal e, assim, o padrão reproduzido será sempre baseado em valores amostrados, pois existem pontos condicionantes. Então, definiu-se que o histograma da imagem de treinamento deveria ser honrado e não foram utilizados dados de amostragem indireta ou regiões. Além disso, foram utilizadas três malhas múltiplas e no mínimo dez repetições em cada malha múltipla. A ponderação das estatísticas dos dados amostrados e padrões é respectivamente 0,6 e 0,4, ou seja, a simulação dará um peso maior a estatística dos dados amostrados do que para a estatística dos padrões da imagem de treinamento e tentará reproduzi-las nestas proporções. A classificação de padrões foi feita com o método de partição cruzada e foram utilizados dez partições primárias e cinco secundárias e o cálculo de distância foi feita através do modelo de pixels.

\section{Base de dados}

Segundo Takafuji (2015), um depósito real não é ideal para o estudo adequado da confiabilidade dos métodos geoestatísticos, uma vez que contornos geológicos em subsuperfície, a posição e os teores exatos dos corpos mineralizados não são conhecidos, afinal, nem todas as camadas são necessariamente aflorantes e a malha de sondagem pode não as interceptar. Para garantir o conhecimento de todo o depósito mineral, ele deve ser estudado e lavrado por inteiro. Para comparar métodos geoestatísticos de simulação, Takafuji (2015) desenvolveu o modelo sintético de um depósito mineral de cobre. A geologia local mimetiza um depósito polimetálico formado por fluidos hidrotermais silicosos que penetraram por uma falha de direção norte-sul e inclinação aproximada de $45^{\circ}$ para leste. A encaixante da mineralização é composta pela intercalação de camadas dobradas de meta-arenito e filitos, gerando resultados em alta variabilidade de padrões geológicos, além da alta variabilidade intrínseca aos depósitos de cobre.

Este depósito foi criado para o estudo de métodos de geoestatística, afinal podem ser feitas sondagens em qualquer local do depósito e assim verificar a confiabilidade dos resultados com a variação da quantidade informação. Para garantir verossimilhança, o modelo geológico foi gerado a partir da simplificação da geologia de um sítio real de mineração e é a interpolação de 2 perfis geológicos semelhantes distantes 600 metros conforme ilustrado na Figura 3.

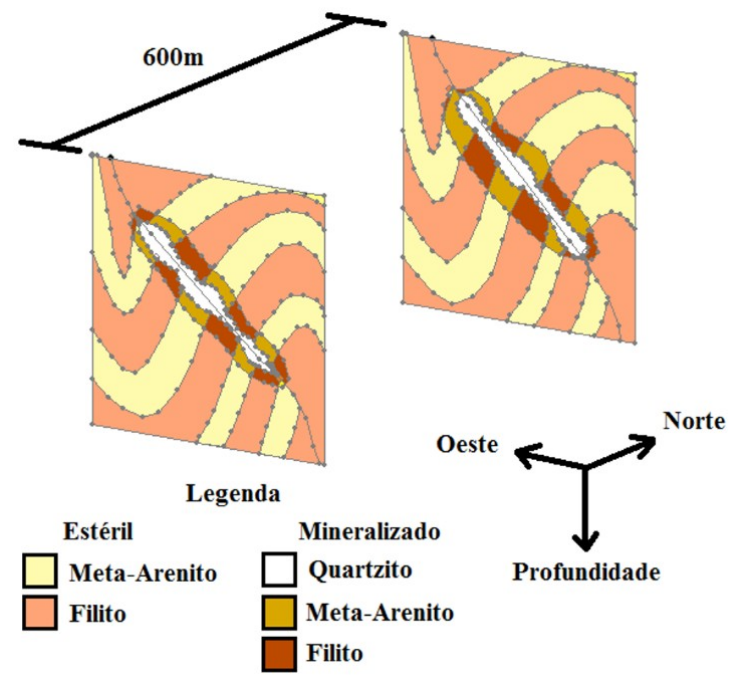

Figura 3 - Contornos do Datamine ${ }^{\circledR}$ que formaram o modelo geológico sem a topografia (fonte: modificada de Takafuji, 2015).

Os blocos dentro da zona mineralizada foram separados em blocos mineralizados - que fazem parte do quartzo remobilizado (zona enriquecida); e blocos não mineralizados- rochas encaixantes constituídas por filito e meta-arenito. Os valores destes blocos foram gerados para a variável cobre por Simulação Sequencial Gaussiana, da qual uma realização foi tomada ao acaso. A simulação não condicional buscou reproduzir um semivariograma real obtido em um depósito equivalente em geologia e variabilidade. Os teores foram gerados dentro da zona mineralizada e fora da mesma utilizando diferentes modelos de semivariogramas (Takafuji 2015).

A imagem de treinamento utilizada possui 30 blocos com dimensões de $10 \mathrm{~m}$ na direção leste, 30 blocos de 
$10 \mathrm{~m}$ na direção norte e 60 blocos de $5 \mathrm{~m}$ na vertical. Esta imagem de treinamento 1 é representada pela região sul do depósito. A área simulada por MPS é contínua à imagem de treinamento, fica à norte e tem 300 metros. Além disto, foram considerados três conjuntos de dados que representam amostragens em malhas regulares separadas a distâncias aproximadas de 60x60 m (Figura 4), 40×40 m (Figura 5) e 20x20 m (Figura 6). Assim, a qualidade das simulações foi analisada em relação ao aumento da quantidade de informações.

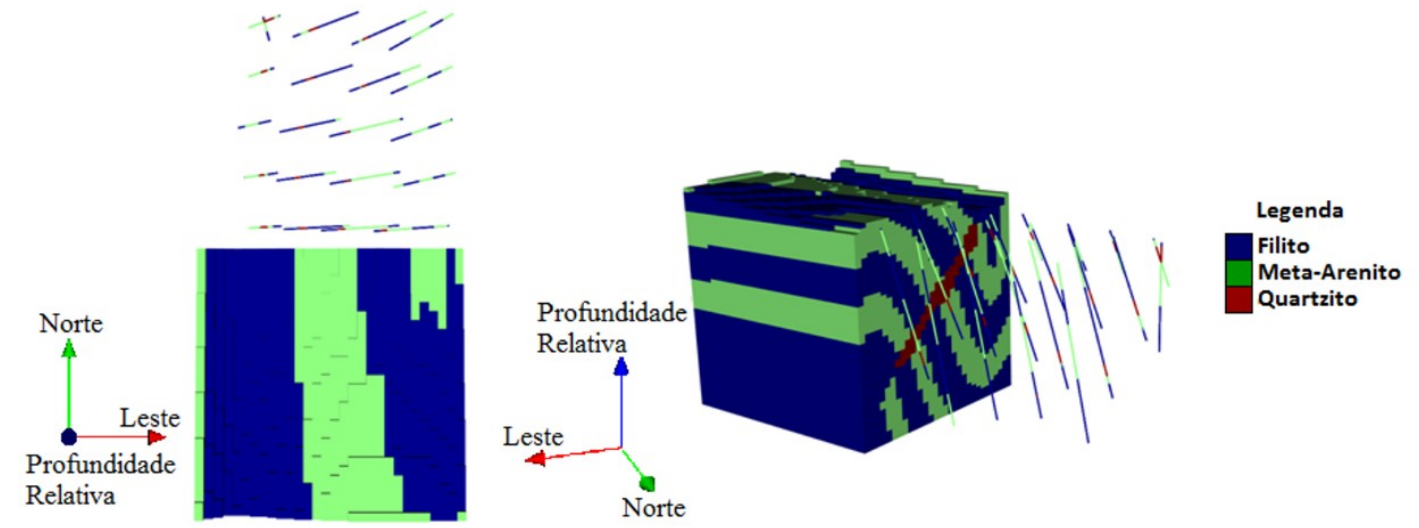

Figura 4- Disposição espacial das amostras coletadas a aproximadamente $60 \mathrm{~m}$ (ao norte) e da imagem de treinamento utilizada à sul.

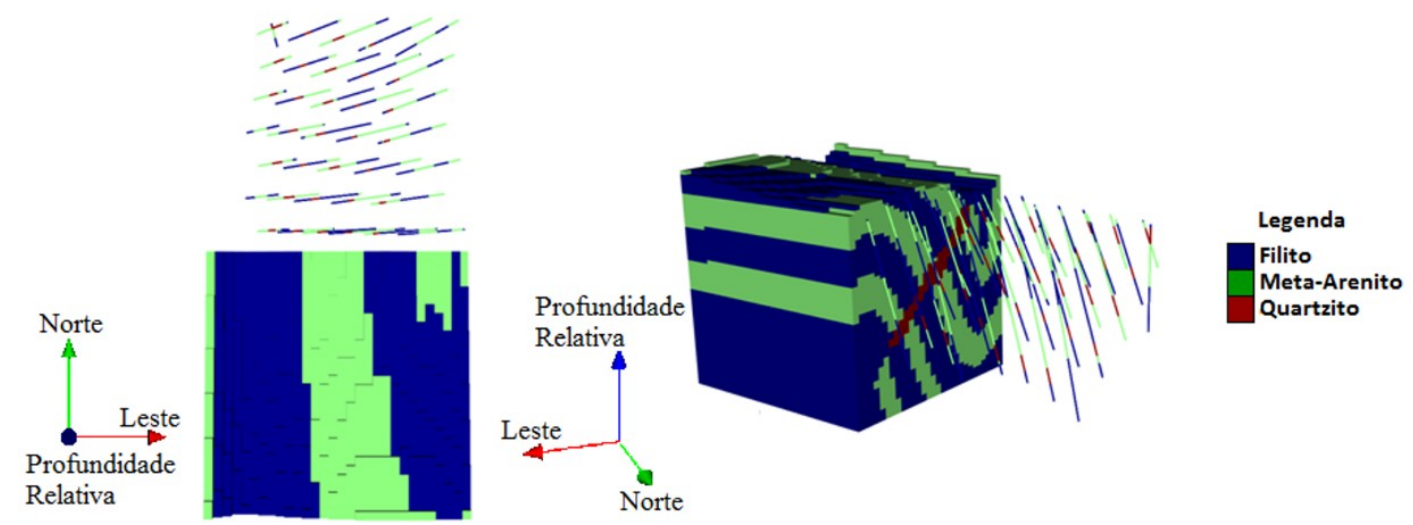

Figura 5- Posição espacial das amostras distanciadas em aproximadamente $40 \mathrm{~m}$ e da imagem de treinamento utilizada.

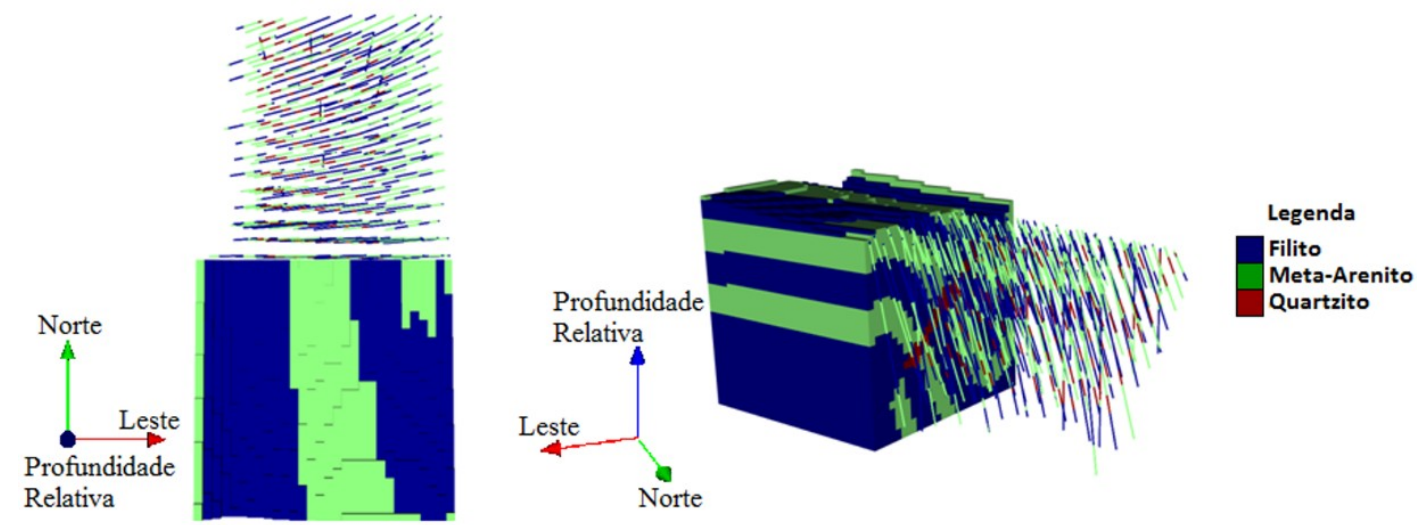

Figura 6- Disposição espacial das amostras distanciadas em aproximadamente $20 \mathrm{~m}$ e da imagem de treinamento utilizada.

\section{Resultados e Comparações}

\subsection{SNESIM}

A partir da amostragem distanciada de $60 \times 60 \mathrm{~m}$ da região norte e com a utilização da imagem de treinamento localizada ao sul, conforme mostrado na Figura 4, foi realizada a simulação pelo método de SNESIM com os parâmetros já citados. Na Figura 7 são apresentadas três realizações, escolhidas ao acaso, da simulação feita com esta amostragem. É possível observar que os padrões não foram reproduzidos de maneira adequada, uma vez que visualmente os resultados não representam satisfatoriamente o modelo geológico esperado. Na primeira imagem, o quartzito está localizado na região da falha, entretanto, sem continuidade evidente, sendo representado em corpos 
descontínuos. Em todas as imagens as intercalações dobradas das camadas parecem lentes de meta-arenito dentro do filito. Apesar da imagem de treinamento fornecer os padrões fidedignos, isto não foi o suficiente para suprir a escassez de dados condicionantes.
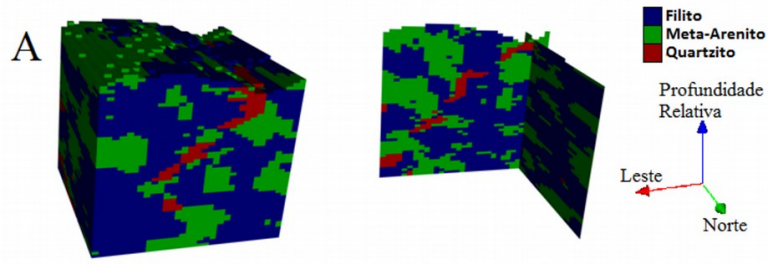

B
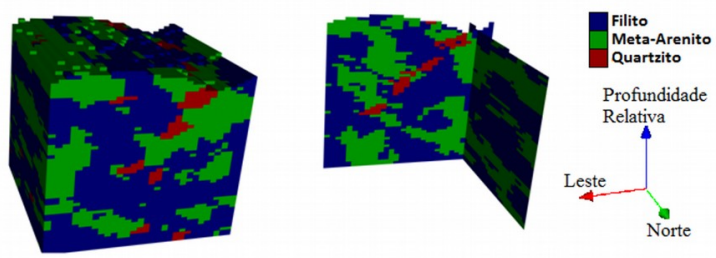

C
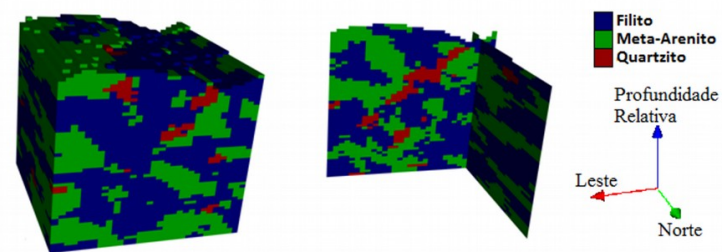

Figura 7- Três realizações, (A), (B) e (C), escolhidas ao acaso da simulação feita a partir da amostragem de 60x60 metros. Do lado esquerdo são todos os blocos simulados e do lado direito são dois perfis em grade para observar o interior do modelo.

$\mathrm{Na}$ Figura 8 são representadas três realizações tomadas ao acaso, da simulação utilizando a amostragem de 40x40 metros. Observa-se que os padrões da imagem de treinamento foram capturados. Pode-se considerar que a região da falha, e os remobilizados silicosos mineralizados, está bem demarcada e posicionada, apesar disso, a continuidade espacial apresenta lacunas. Como as amostras condicionais são mais próximas diminui a possibilidade de padrões diferentes serem estimados na simulação. O filito e o meta-arenito (respectivamente em azul e verde na Figura 8) apresentam os padrões esperados com algumas imperfeições nos contornos, porém, estão melhor definidos quando comparados com os resultados apresentados na Figura 7. Nas porções onde não há amostras - como na região oeste em cotas menores - os resultados não reproduzem o esperado, pois esta é uma porção do domínio com pequena quantidade de dados condicionantes.

Apresentam-se na Figura 9 três realizações desta simulação, nas três imagens é possível observar que a continuidade espacial do corpo foi reproduzida realisticamente, portanto a técnica testada pode ser considerada adequada. Nas regiões onde há poucas amostras (dados condicionantes), como por exemplo na porção de baixa cota a nordeste da imagem $\mathrm{C}$, a não reprodução do padrão da imagem de treinamento pode ser observada. Uma vez que não é interessante realizar sondagem além do corpo de minério, também não é considerado o estudo detalhado desta região.
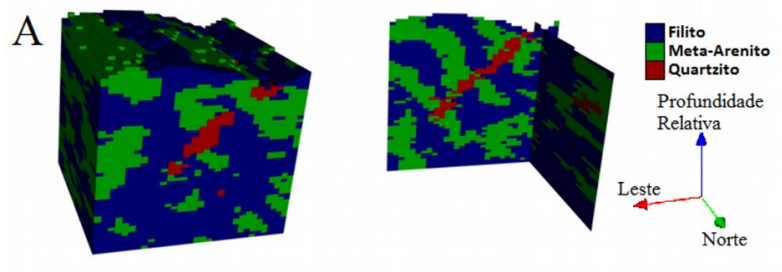

B
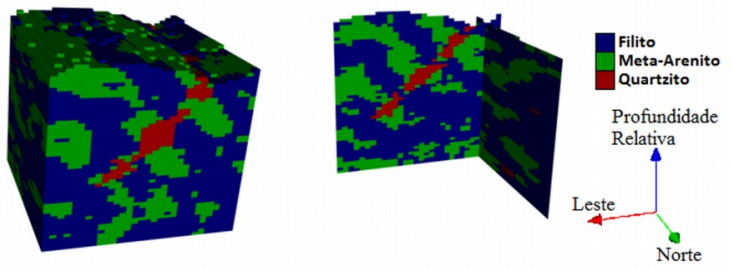

C
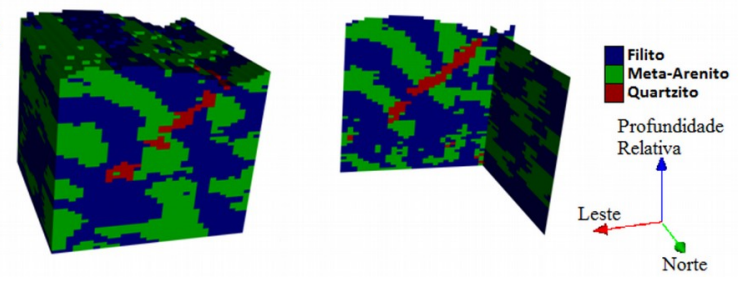

Figura 8- Três realizações da simulação feita com os dados coletados segundo a malha de $40 \times 40$ metros.
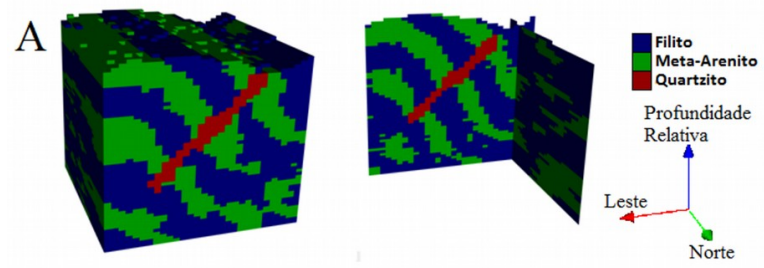

B
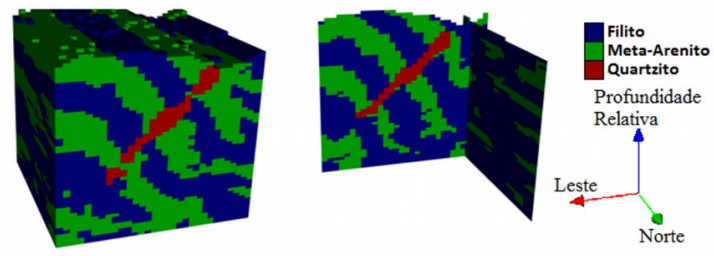

C
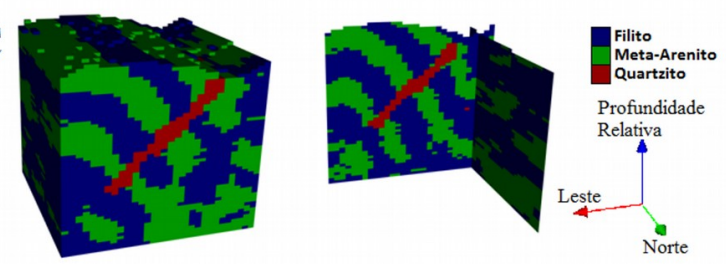

Figura 9- Três realizações da simulação feita utilizando uma amostragem de $20 \times 20$ metros.

\subsection{FILTERSIM}

As mesmas bases de dados, descritas anteriormente e ilustradas nas Figuras 5, 6 e 7 foram utilizadas na simulação FILTERSIM. O objetivo desta escolha foi poder comparar os resultados obtidos por ambos os métodos. Na Figura 10 são apresentados os resultados de três realizações, também escolhidas aleatoriamente, da simulação com base na amostragem de 60x60 metros. 
A

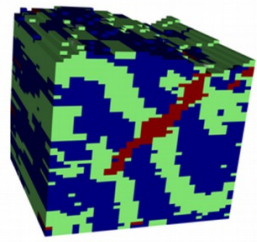

B

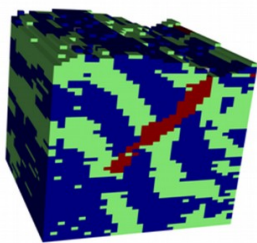

$\mathrm{C}$

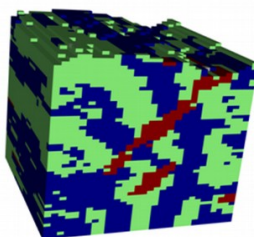

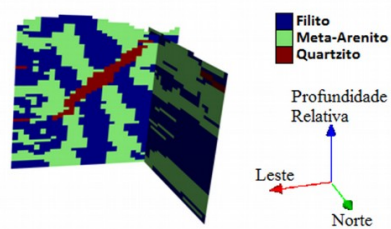
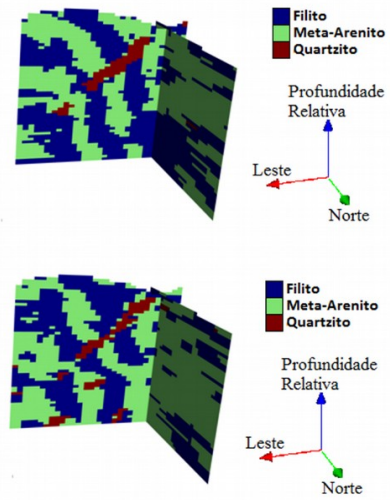

Figura 10- Resultado de três realizações, escolhidas aleatoriamente, feitas com amostras condicionais separadas de 60 em 60 metros.

Por análise visual, o resultado do FILTERSIM apresenta estruturas mais parecidas com o que se observa na imagem de treinamento quando comparada com o resultado apresentado pelo SNESIM (Figura 7). A Figura 11, representa três realizações do FILTERSIM para a amostragem de 40 metros. Conforme se observa, as porções centrais e superiores são as com resultado mais próximo ao presente na imagem de treinamento. O quartzito encontra-se, em geral, menos contínuo e menor do que o esperado, enquanto na porção sul a continuidade espacial se perde. Em comparação com o SNESIM (Figura 8), o FILTERSIM apresenta resultados visualmente melhores.

A

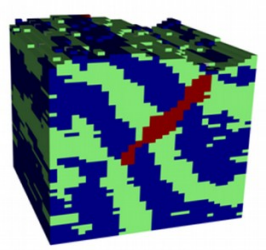

B

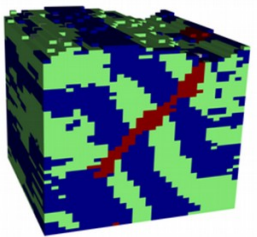

C

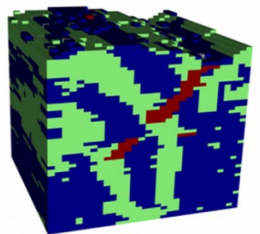

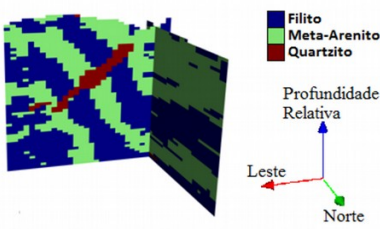
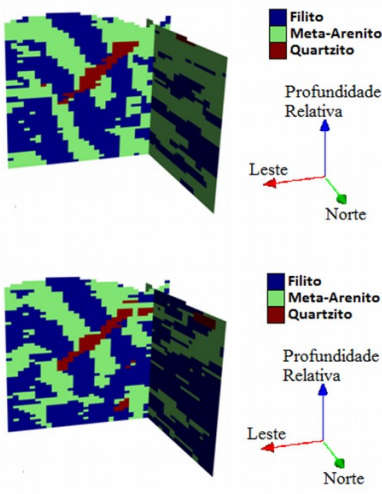

Profundid
Relativa Leste

Figura 11- Resultado de três realizações diferentes feitas com amostras condicionais separadas de 40 em 40 metros.

Na Figura 12 apresenta-se o resultado da simulação, por FILTERSIM com as sondagens distanciadas em 20 metros. O resultado desta simulação reproduziu a configuração básica presente na imagem de treinamento de forma evidente, exceto na porção superficial à oeste onde podem ser observados viéses nos resultados devido à falta de informação nesta região. Além deste melhor resultado a análise da Figura 12C mostra que o quartzito pode ser simulado em regiões inesperadas, pela ausência de informação condicionante em um método sequencial. Porém, na média das simulações, isto é apenas um ruído.
A

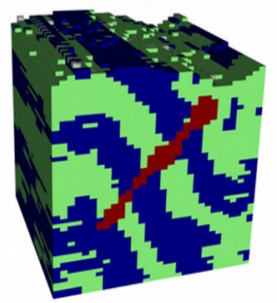

B

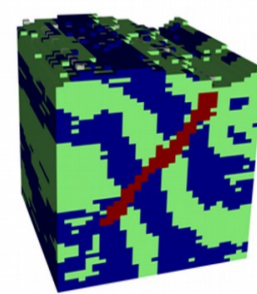

$\mathrm{C}$

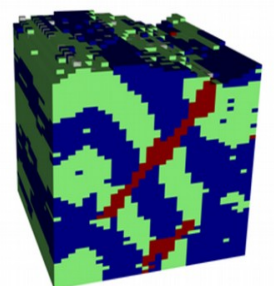

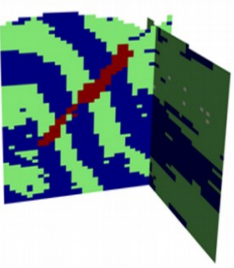
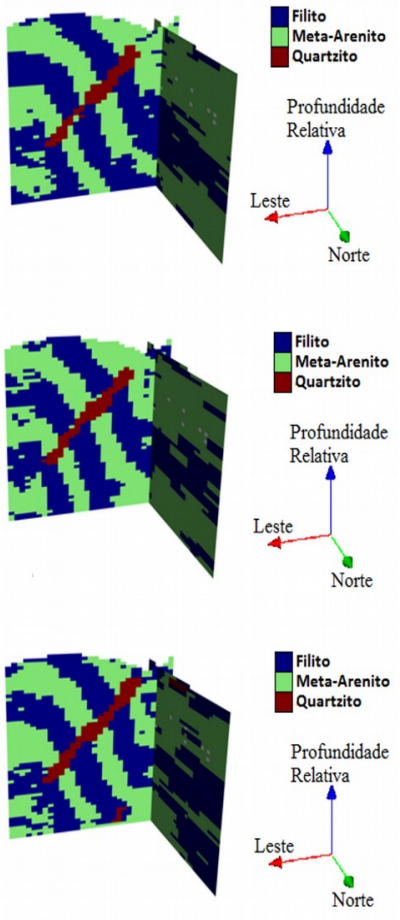

Figura 12- Resultado de três realizações diferentes feitas com amostras condicionais separadas de $20 \mathrm{em} 20$ metros.

A Tabela 1 apresenta a relação entre as porcentagens de cada litotipo, comparando a imagem de treinamento, a amostragem e o resultado médio das simulações por FILTERSIM e por SNESIM. Nesta tabela fica evidente a influência da imagem de treinamento nestes métodos de simulação, pois os dados estatísticos se aproximam mais da imagem de treinamento que da amostragem. Enquanto, a Tabela 2 corrobora a análise visual mostrando que as estatísticas do FILTERSIM se aproximam mais da imagem de treinamento que as do SNESIM. E, na Tabela 3, o método SNESIM apresentou estatísticas descritivas semelhantes àquelas da imagem de treinamento. 
Tabela 1- Análise quantitativa da imagem de treinamento, dos dados amostrados e de todas as realizações, do SINESIM e FILTERSIM, com a amostragem de 60 metros, em média.

\begin{tabular}{lllll}
\hline Categorias & Imagem & Amostra & SINESIM & FILTERSIM \\
\hline Filito & $54 \%$ & $51 \%$ & $57 \%$ & $53 \%$ \\
\hline Meta-renito & $41 \%$ & $40 \%$ & $38 \%$ & $43 \%$ \\
\hline Quartzito & $5 \%$ & $9 \%$ & $4 \%$ & $5 \%$ \\
\hline
\end{tabular}

Tabela 2- Análise quantitativa da imagem de treinamento, dos dados amostrados e de todas as realizações, do SINESIM e FILTERSIM, com a amostragem distante 40 metros, em média.

\begin{tabular}{lllll}
\hline Categorias & Imagem & Amostra & SINESIM & FILTERSIM \\
\hline Filito & $54 \%$ & $45 \%$ & $58 \%$ & $52 \%$ \\
\hline $\begin{array}{l}\text { Meta- } \\
\text { Arenito }\end{array}$ & $41 \%$ & $44 \%$ & $37 \%$ & $43 \%$ \\
\hline Quartzito & $5 \%$ & $11 \%$ & $5 \%$ & $6 \%$ \\
\hline
\end{tabular}

Tabela 3- Análise quantitativa da imagem de treinamento, dos dados amostrados e de todas as realizações, do SINESIM e FILTERSIM, com a amostragem distante 20 metros, em média.

\begin{tabular}{lllll}
\hline Categorias & Imagem & Amostra & SINESIM & FILTERSIM \\
\hline Filito & $54 \%$ & $45 \%$ & $57 \%$ & $50 \%$ \\
\hline $\begin{array}{l}\text { Meta- } \\
\text { Arenito }\end{array}$ & $41 \%$ & $43 \%$ & $37 \%$ & $45 \%$ \\
\hline Quartzito & $5 \%$ & $12 \%$ & $6 \%$ & $5 \%$ \\
\hline
\end{tabular}

\section{Considerações finais}

Os métodos de simulação de multiponto ainda necessitam estudos adicionais para se consolidarem entre os mais utilizados. Porém, a inclusão deles em programas como SGeMS® e Isatis ${ }^{\circledR}$ ajudam a aumentar sua difusão. Em questões práticas, as dificuldades encontradas são referentes a definição da imagem de treinamento e definição do modelo e das proporções entre estatísticas descritivas dos dados e da imagem de treinamento, os quais se comparam, respectivamente, com o estudo do variograma (experimental e modelo teórico) e com a definição dos parâmetros de vizinhança de busca. A maior crítica a estes métodos diz respeito à criação/obtenção da uma imagem de treinamento, pois na mineração, a depender da fase que se está no empreendimento minerário, não há informação suficiente que permita defini-la adequadamente. Quanto mais detalhada é a imagem de treinamento, mais adequados são os resultados e o detalhamento ideal requerido pelas técnicas, muitas vezes, são obtidas apenas em fase avançada da lavra. A utilização de afloramentos/depósitos análogos é uma possibilidade que atenua o problema de escolha da imagem de treinamento adequada, mas não o elimina, uma vez que há a necessidade de garantir a existência de todos os padrões esperados no depósito e, por isso, ele precisa ser bem conhecido.

Os resultados encontrados nesta comparação mostram que o método FILTERSIM consegue representar adequadamente a continuidade espacial, mesmo para as amostragens de baixa densidade. Enquanto isto, o método SNESIM não reproduz esta continuidade quando os pontos condicionantes são distantes (amostragem de $60 \mathrm{~m}$ e $40 \mathrm{~m}$ ). Este método apresentou mais ruído nos resultados. Além disto, estatisticamente, o FILTERSIM foi melhor para as bases de dados com amostras mais distantes (60 metros e 40 metros) e o SNESIM para amostras separadas por 20 metros. Ambos os métodos valorizaram mais a proporção da imagem de treinamento que a da amostragem.

\section{Agradecimentos}

Os autores agradecem à CAE Mining pela cessão da licença do programa Studio $3 \circledR$. Assim como, ao laboratório COSMO da Universidade McGill no Canadá pelo apoio com os métodos estudados. À CAPES (Comissão de Aperfeiçoamento de Pessoal do Nível Superior) pelas bolsas de doutorado concedidas.

\section{Referências bibliográficas}

ARPAT B. 2005. Sequential Simulation with patterns. Doctor Dissertation. Department of Petroleum Engineering, Stanford University, 166p.

ARPAT B., CAERS J. 2005 A multiple scale, pattern-based approach to sequential simulation. In: LEUANGTHONG O.,
DEUTSCH C. V. (eds) Geostatistics Banff 2004. Springer, Dordrecht, $255-264 \mathrm{p}$.

ARPAT B., CAERS J. 2007 Conditional simulation with patterns. Mathematical Geology, 39(2):177-203. 
CAERS J., ZHANG T. 2002. Multiple-point geostatistics: a quantitative vehicle for integration geologic analogs into multiple reservoir model, Integration of outcrop and modern analog data in reservoir models. Stanford University, Stanford Center for Reservoir Forecasting. 24p. (Internal Report)

DIMITRAKOPOULOS R., MUSTAPHA H., GLOAGUEN E. 2010 High-order statistics of spatial random fields: exploring spatial cumulants for modelling complex, non-Gaussian and non-linear phenomena. Mathematical Geosciences, 42:65-99.

GLOAGUEN E., DIMITRAKOPOULOS R. 2009 Two-dimensional conditional simulations based on the wavelet decomposition of training images. Mathematical Geosciences, 41(6) 679-701.

GUARDIANO F., SRIVASTAVA M. 1993 Multivariate Geostatistics: Beyond Bivariate Moments. In: SOARES A. (ed) Geostatistics-Tróia '92. Kluwer Academic Publishers, Dordrecht, 133-144 p.

HONARKHAH M., CAERS J. 2010 Stochastic Simulation of Patterns Using Distance-Based Pattern Modeling. Mathematical Geosciences, 42(5):487-517.

JOURNEL A., ZHANG T. 2006 The necessity of a Multiple-Point Prior Model. Mathematical Geology, 38(5):591-610.

LYSTER S., DEUTSCH C.V., ORTIZ J.M. 2004 Shot Note: Some Implementation Aspects of Multiple-Point Simulation. University of Alberta, CCG Annual Report Papers. 6p. (Internal Report)

MARIETHOZ G., CAERS J. 2014. Multiple-point Geostatistics: Stochastic Modeling with Training Images. John Wiley \& Sons, Chichester, UK $376 \mathrm{p}$.

MARIETHOZ G., RENARD P., STRAUBHAAR J. 2010. The Direct Sampling Method to Perform Multiple-Point Geostatistical Simulations. Water Resources Research, 46(11).

ORTIZ J.M. 2003 Selected Aspects of Multiple-Point Statistics. University of Alberta, CCG Annual Report Papers. 30p. (Internal Report)

REMY N., BOUCHER A., WU J. 2011 Applied Geostatistics with SGeMS - A User's Guide. Cambridge University Press, Cambridge, 264p.

STREBELLE S. 2002 Conditional Simulation of Complex Geological Structures Using Multiple-Point Statistics. Mathematical Geology, 34(1):1-21.

TAKAFUJI E.H.M. 2015 Estudo Comparativo Entre a Simulação Sequencial Gaussiana e a Simulação Baseada em Wavelets Aplicado a Quantificação de Minério de $\mathrm{Cu}$ em um Depósito Sintético. Dissertação de Mestrado. Pós-Graduação em Recursos Minerais e Hidrogeologia. Instituto de Geociências, Universidade de São Paulo, 102p.

VRIES L. M., CARRERA J., FALIVENE O., GRATACÓS O., SLOOTEN L.J. 2009 Application of Multiple Point Geostatistics to Non-stationary Images. Mathematical Geosciences, 41:29-42.

WU J., ZHANG T., JOURNEL A. 2008 Fast FILTERSIM Simulation with Score-based Distance. Mathematical Geosciences, 40(7):773-788.

ZHANG T., SWITZER P., JOURNEL A. 2006 Filter-Based Classification of Training Image Patterns for Spatial Simulation. Mathematical Geology, 38(1):63-80.

Manuscrito ID 54132

Submetido em julho de 2017 Aceito em dezembro de 2017 